\title{
Hedgehog Inhibitors Suppress Osteoclastogenesis in In Vitro Cultures, and Deletion of Smo in Macrophage/Osteoclast Lineage Prevents Age-Related Bone Loss
}

\author{
Yukihiro Kohara ${ }^{1, *}$, Ryuma Haraguchi ${ }^{1, *}$, Riko Kitazawa ${ }^{1,2}$, Yuuki Imai ${ }^{3,4,5}$ and \\ Sohei Kitazawa ${ }^{1}$ \\ 1 Department of Molecular Pathology, Ehime University Graduate School of Medicine, Shitsukawa, Toon City, \\ Ehime 791-0295, Japan \\ 2 Division of Diagnostic Pathology, Ehime University Hospital, Shitsukawa, Toon City, Ehime 791-0295, Japan \\ 3 Division of Integrative Pathophysiology, Proteo-Science Center, Ehime University Graduate School of \\ Medicine, Shitsukawa, Toon City, Ehime 791-0295, Japan \\ 4 Division of Laboratory Animal Research, Advanced Research Support Center, Ehime University, Toon, \\ Ehime 791-0295, Japan \\ 5 Department of Pathophysiology, Ehime University Graduate School of Medicine, Toon, \\ Ehime 791-0295, Japan \\ * Correspondence: kohara.yukihiro.yu@ehime-u.ac.jp (Y.K.); ryumaha@m.ehime-u.ac.jp (R.H.); \\ Tel.: +81-89-960-5265 (Y.K. \& R.H.)
}

Received: 24 March 2020; Accepted: 14 April 2020; Published: 15 April 2020

\begin{abstract}
The functional role of the Hedgehog (Hh)-signaling pathway has been widely investigated in bone physiology/development. Previous studies have, however, focused primarily on $\mathrm{H} h$ functions in bone formation, while its roles in bone resorption have not been fully elucidated. Here, we found that cyclopamine (smoothened (Smo) inhibitor), GANT-58 (GLI1 inhibitor), or GANT-61 (GLI1/2 inhibitor) significantly inhibited RANKL-induced osteoclast differentiation of bone marrow-derived macrophages. Although the inhibitory effects were exerted by cyclopamine or GANT-61 treatment during $0-48 \mathrm{~h}$ (early stage of osteoclast differentiation) or 48-96 h (late stage of osteoclast differentiation) after RANKL stimulation, GANT-58 suppressed osteoclast formation only during the early stage. These results suggest that the Smo-GLI1/2 axis mediates the whole process of osteoclastogenesis and that GLI1 activation is requisite only during early cellular events of osteoclastogenesis. Additionally, macrophage/osteoclast-specific deletion of Smo in mice was found to attenuate the aging phenotype characterized by trabecular low bone mass, suggesting that blockage of the Hh-signaling pathway in the osteoclast lineage plays a protective role against age-related bone loss. Our findings reveal a specific role of the Hh-signaling pathway in bone resorption and highlight that its inhibitors show potential as therapeutic agents that block osteoclast formation in the treatment of senile osteoporosis.
\end{abstract}

Keywords: osteoclasts; Hedgehog signaling; cyclopamine; GANT-58; GANT-61; Smoothened; GLI1; GLI2

\section{Introduction}

Hedgehog (Hh) signaling, well-known as a mitogen and morphogen during animal development, also regulates adult tissue homeostasis and tumorigenesis [1-4]. Hh ligands in mammals are three proteins, Indian hedgehog (Ihh), Sonic hedgehog, and Desert hedgehog; although they are expressed in different cells and tissues, their functions are not considered different $[5,6]$. Hh ligands bind to and inhibit main receptor Patched-1 (PTCH1, encoded by the Ptch1 gene), permitting the activation of 
Hh signal transducer smoothened (SMO, encoded by the Smo gene) and transmitting intracellular signaling through transcription factors of the GLI family $[5,7,8]$. Among GLI transcription factors GLI1, GLI2, and GLI3 that collectively mediate all Hh pathways, GLI2 and GLI3 are the initial mediators of Hh signal transduction, and GLI1, being a direct target gene, functions as a positive feedback to enhance GLI activity [8]. GLI1 acts as a positive transcriptional effector, while GLI2 and GLI3 function predominantly as transcriptional activators or repressors in a cellular context-dependent manner. In the activated Hh-signaling pathway, GLI proteins are released from the inhibitory complex with the Suppressor of Fused (SuFu) $[9,10]$. Finally, activated GLI forms are translocated to the nucleus, where they act as transcription factors and promote Hh target gene expression.

Agents that specifically and selectively target the Hh-signaling pathway are available for experiments [11-16]. Cyclopamine is a bioactive steroidal alkaloid extracted from natural plants, and its synthetic compounds inhibit SMO function by direct interaction with SMO-transmembrane domains [14,15]. GANT-58 and GANT-61 are identified as small-molecule inhibitors of GLI proteins [11,13]. GANT-58 prevents GLI1-dependent transcription through the inhibition of its post-translational modification [11]. In contrast, GANT61 blocks GLI1/DNA interaction by direct binding to the GLI1 protein and impairs GLI2-mediated transcription [11,13]. The GANT61-binding element shows a high degree of sequence homology between GLI1 and GLI2, making GANT61 an inhibitor of both GLI1- and GLI2-induced transcriptions [13]. At present, targeting Hh signaling by inhibitors, including cyclopamine and GANTs, has been drawing attention as a potential therapeutic strategy in various human diseases.

The Hh-signaling pathway contributes to skeletal development, bone homeostasis, and the progression of tumor bone metastasis. During endochondral ossification, Ihh produced by hypertrophic chondrocytes stimulates osteoblastic bone formation by promoting the expression of Runx2, which is known as a master transcription factor for osteoblast differentiation $[2,17,18]$. The study of Rodda and McMahon revealed that Hh signaling is not required in the early differentiation phase of an osteoblast for further osteoblast maturation [19]. In mature osteoblasts of adult mice, activated Hh signaling, caused by a deficiency in PTCH1, leads to low bone strength, with reduced bone density attributed to enhanced osteoclast-induced bone resorption [20]. Consistent with this, Hh-signaling inhibition by mature osteoblasts' specific conditional ablation of Smo results in protection from bone loss in one-year-old mice [20]. By contrast, a low level activation of Hh signaling, caused by PTCH1 haploinsufficiency, enhances osteoblast differentiation and increases bone mass [21]. During osteolytic cancer bone metastasis, augmented GLI activity in tumor cells leads to secretion of parathyroid hormone-related protein (PTHrP), which induces the Receptor Activator of Nuclear factor- $\mathrm{kB}$ Ligand (RANKL) expression in osteoblasts, thus promoting osteoclastogenesis [22]. These studies have, however, attached importance mainly to the Hh function on osteogenic lineage cells, the specific or direct role of Hh signaling on osteoclastic bone resorption being unknown.

Osteoclasts, differentiated from the monocyte/macrophage lineage stimulated by RANKL, destroy the bone matrix and stimulate osteoblast differentiation and bone formation, thus maintaining bone remodeling $[23,24]$. Interference of osteoclastic bone resorption is a therapeutic target of anti-osteoporosis drugs, such as bisphosphonates and the anti-RANKL antibody (denosumab) [25,26]. Oral administration of cyclopamine increases bone mass because of the reduced bone resorption in mice, suggesting that cyclopamine can also be a therapeutic drug for osteoporosis [27]. Yet, the mechanism of the inhibitory effect of cyclopamine on bone resorption is not fully understood.

Here, we show that treatment with cyclopamine, GANT-58, or GANT-61 exerts a potent inhibitory effect on osteoclast formation in primary cultured bone marrow-derived macrophages (BMMs) stimulated by RANKL and suggest that Hh signaling is a requisite for osteoclastic differentiation. Moreover, macrophage/osteoclast lineage-specific Smo gene deficiency protected from age-related bone loss. Thus, we provide evidence that Hh signaling in the macrophage/osteoclast lineage mediates osteoclastogenesis in vitro and in vivo. 


\section{Results}

\subsection{Changes in Expression of Hedgehog $(\mathrm{Hh})$ Signaling-Related Genes during Osteoclast Differentiation}

First examined were the changes in the expression of Hh signaling-related genes during osteoclast differentiation. Primary cultured bone marrow-derived macrophages (BMMs) differentiated into mature osteoclasts (mOC) $96 \mathrm{~h}$ after RANKL stimulation (Figure 1A). Quantitative real-time RT-PCR (qRT-PCR) analysis revealed that Ctsk mRNA expression significantly increased after RANKL stimulation (Figure 1B), suggesting a progressing of osteoclastic differentiation. No obvious change in Gli2 and Gli3 mRNA expression, whereas Smo and Gli1 mRNA expression decreased gradually with osteoclastic differentiation, as assessed by qRT-PCR (Figure 1C). On the other hand, Ptch1 mRNA expression significantly increased gradually with osteoclastic differentiation (Figure 1C). These results suggest that $\mathrm{Hh}$ signaling in the macrophage/osteoclast lineage is associated with osteoclastic differentiation.

A

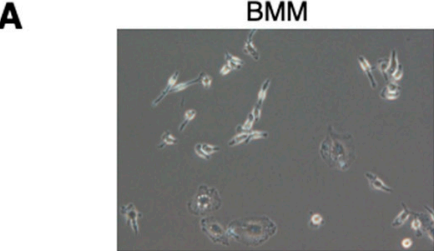

RANKL (h)
0

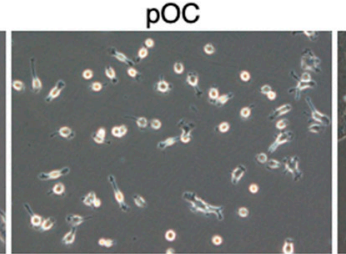

48
$\mathrm{mOC}$

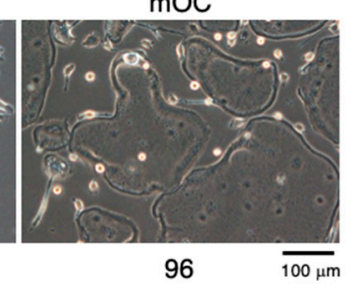

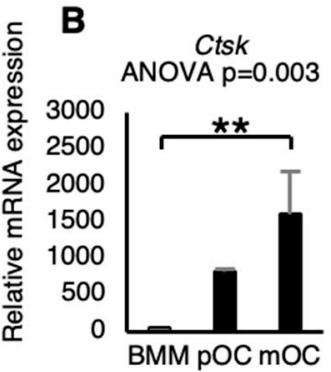

C
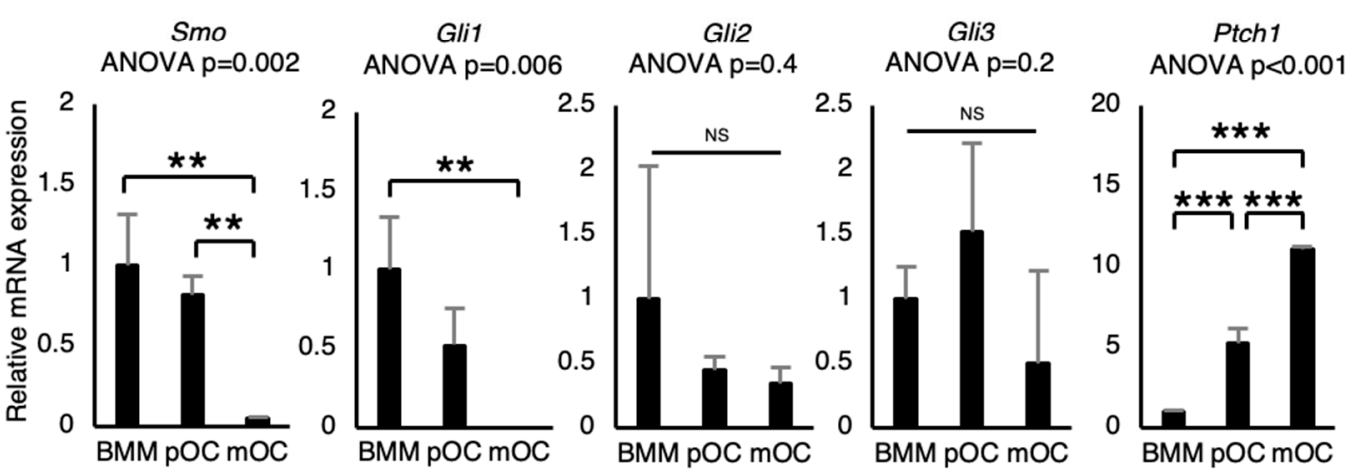

Figure 1. Changes in expression of Hedgehog $(\mathrm{Hh})$ signaling-related genes during osteoclast differentiation. (A) Representative microscopic images of bone marrow-derived macrophages (BMMs: without RANKL), pre-osteoclasts (pOC: $48 \mathrm{~h}$ after RANKL stimulation), or mature osteoclasts (mOC: $96 \mathrm{~h}$ after RANKL stimulation). (B) Ctsk mRNA expression was determined by quantitative real-time RT-PCR $(n=3)$. (C) Hh signaling-related gene expression levels were determined by quantitative real-time RT-PCR $(n=3)$. The abundance of target mRNA was normalized by that of Actb mRNA. Data are represented as means \pm s.d. One-way ANOVA, followed by Tukey-Kramer's test; NS, not significant; ${ }^{* *} p<0.01 ;{ }^{* * *} p<0.001$.

\subsection{Cyclopamine Suppresses Osteoclastogenesis}

Since treatment with SMO inhibitor cyclopamine increases trabecular bone volume because of reduced bone resorption in mice [27], the effect of cyclopamine treatment on primary osteoclastic cultures was examined. Treatment with cyclopamine significantly decreased formation of $\mathrm{TRAP}^{+}$multinuclear cells (MNCs) in a dose-dependent manner (Figure 2A). Although no recombinant Hh ligand proteins were added to the osteoclastic cultures, cyclopamine treatment suppressed osteoclastogenesis. Possibly, therefore, Hh ligands contained in FBS may be important for osteoclast formation in vitro. That the addition of recombinant Sonic hedgehog enhances osteoclast formation in RAW264.7 cells [28] suggests that Hh ligands mediate osteoclastogenesis. Next, to investigate when it suppresses osteoclast differentiation, cyclopamine treatment was implemented over terms of 0-48 h, 48-96 h, and 0-96 h after 
RANKL stimulation. The outcome disclosed that the suppression was induced during all terms, as assessed by TRAP staining and a TRAP activity assay (Figure 2B). Moreover, quantitative real-time RT-PCR analysis revealed that treatment with cyclopamine significantly decreased osteoclastic marker genes Tnfrsf11a (encoding RANK), Ctsk, Acp5 (encoding TRAP), Nfatc1, and Dcstamp without affecting Calcr (encoding Calcitonin receptor) at $96 \mathrm{~h}$ after RANKL stimulation (Figure 2C). These results suggest that cyclopamine induces inhibitory effects at all stages of osteoclastic differentiation.

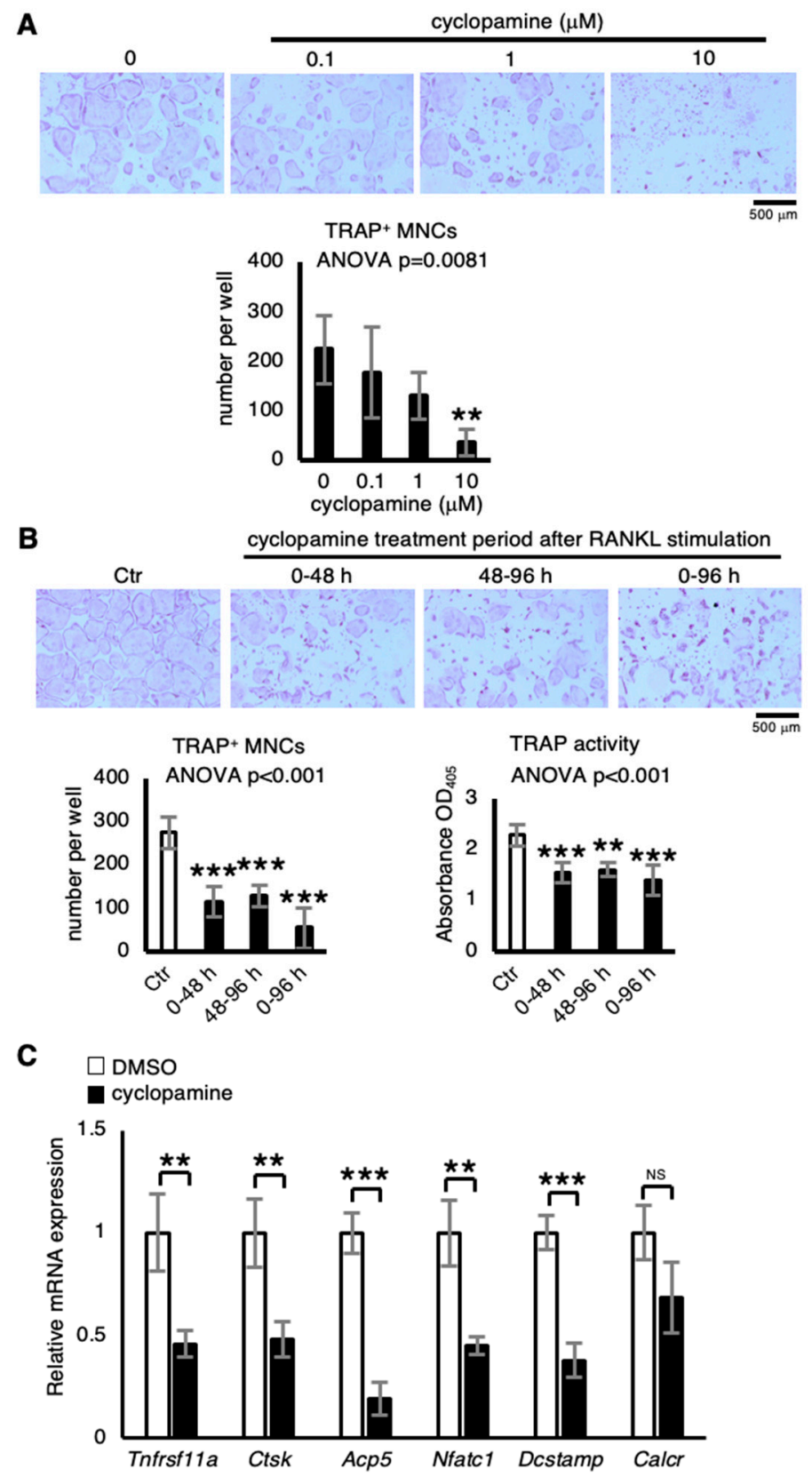

Figure 2. Cyclopamine suppresses osteoclastogenesis. (A) The effect of cyclopamine on osteoclast formation. The number of $\mathrm{TRAP}^{+}$multinuclear cells (MNCs) were counted $(n=4)$. (B) Osteoclastogenic cultures treated with $10-\mu \mathrm{M}$ cyclopamine throughout the $96 \mathrm{~h}$ period $(0-96 \mathrm{~h})$ or only during the first $\left(0-48 \mathrm{~h}\right.$ ) or second period (48-96 h); the number of $\mathrm{TRAP}^{+}$multinuclear cells (MNCs) and TRAP activity determined $96 \mathrm{~h}$ after RANKL stimulation $(n=4)$. (C) Effect of cyclopamine treatment on osteoclastic gene expression in osteoclastic cultures $(n=3)$. Abundance of target mRNA normalized by that of Actb mRNA. Data are represented as means \pm s.d. One-way ANOVA, followed by Dunnett's test $(\mathbf{A}, \mathbf{B})$ or unpaired two-sided Student's $t$-test (C); NS, not significant; ${ }^{* *} p<0.01$ and ${ }^{* * *} p<0.001$. 


\subsection{Other Hh Signaling Inhibitors that Suppress Osteoclastogenesis}

Next examined was the effect of other Hh signaling inhibitors, GANT-58 (GLI1 inhibitor) and GANT-61 (GLI1/2 inhibitor), on osteoclast differentiation. Treatment with GANT-58 and GANT-61 significantly decreased TRAP ${ }^{+}$MNC formation in a dose-dependent manner, as did the treatment with cyclopamine (Figure 3A), strongly suggesting that Hh signaling in the macrophage/osteoclast lineage is requisite for osteoclast formation. Interestingly, GANT-58 treatment during the first 48 -h term $(0-48 \mathrm{~h})$ was sufficient to inhibit osteoclastogenesis but not during the second term (48-96 h) (Figure 3B). On the other hand, GANT-61 treatment exerted an inhibitory effect on osteoclast differentiation during the three terms, as did cyclopamine treatment (Figure 3C). These results suggest that GLI1 and GLI2 activation is essential for the early and late stages, respectively, of osteoclast differentiation.
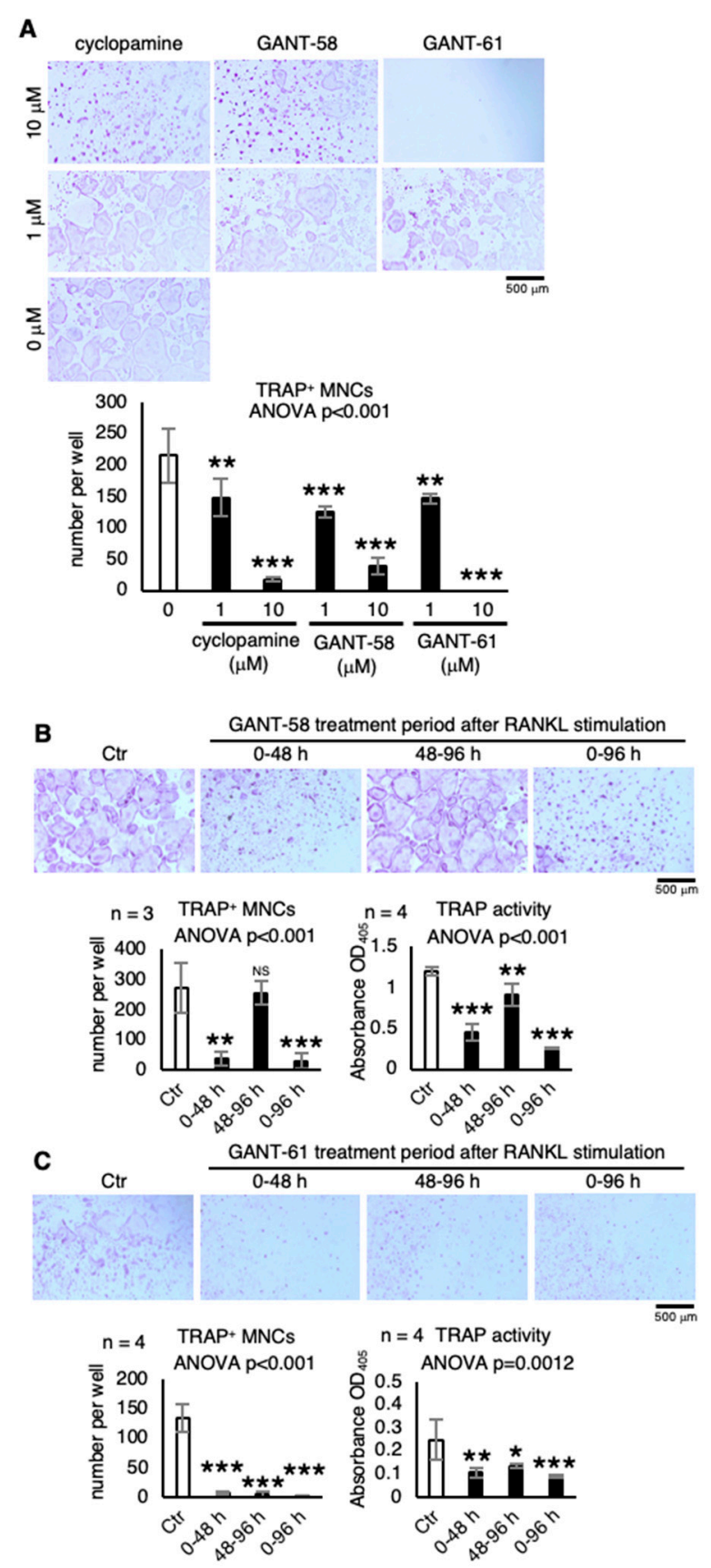

Figure 3. Other Hh signaling inhibitors that suppress osteoclastogenesis. (A) Effect of Hh signaling inhibitors cyclopamine, GANT-58, and GANT-61 on osteoclast formation. The number of TRAP ${ }^{+}$ 
multinuclear cells (MNCs) were counted $(n=3)$. (B,C) Osteoclastogenic cultures treated with $(\mathbf{B})$ 10- $\mu$ M GANT-58 or (C) 5- $\mu$ M GANT-61 throughout the 96-h period (0-96 h) or only during the first $\left(0-48 \mathrm{~h}\right.$ ) or second period (48-96 h); the number of TRAP ${ }^{+}$multinuclear cells (MNCs) and TRAP activity determined at $96 \mathrm{~h}$ after RANKL stimulation $(n=3-4)$. Data are represented as means \pm s.d. One-way ANOVA, followed by Dunnett's test; NS, not significant; ${ }^{*} p<0.05,{ }^{* *} p<0.01$, and ${ }^{* * *} p<0.001$.

\subsection{Hh Signaling Inhibitors Suppress Osteoclastic Viability}

MTT cell viability assay carried out to investigate the effect of Hh signaling inhibitors on osteoclast growth revealed that RANKL stimulation increased the number of BMMs and that Hh inhibitors cyclopamine, GANT-58, and GANT-61 inhibited osteoclastic viability (Figure 4), suggesting that Hh signaling mediates osteoclastic viability.

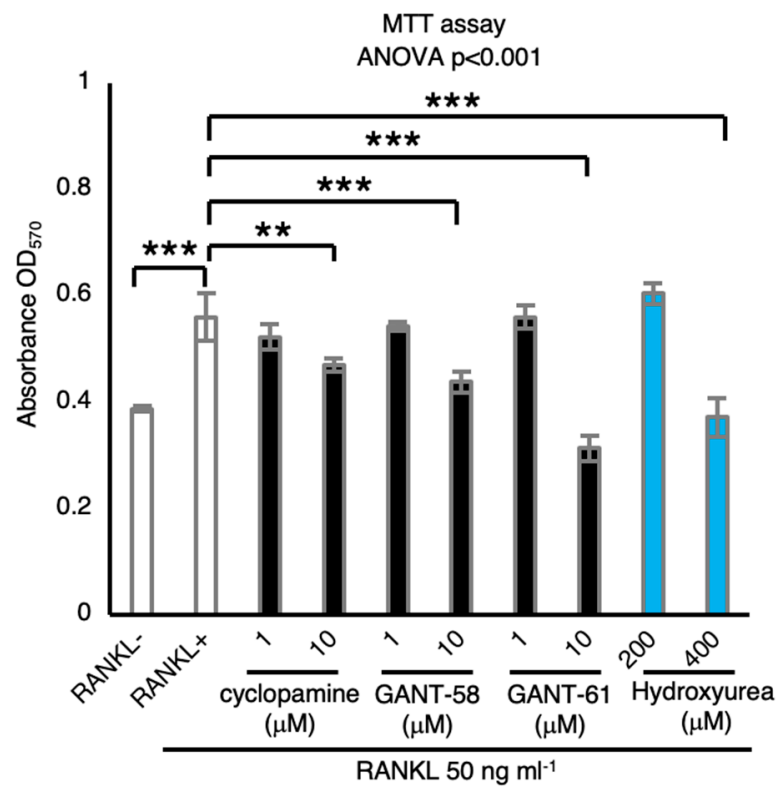

Figure 4. Hh signaling inhibitors suppress osteoclastic viability. The effect of Hh signaling inhibitors on osteoclast viability determined by MTT assay $(n=3)$. Hydroxyurea used as DNA synthesis inhibitor. Data are represented as means \pm s.d. One-way ANOVA, followed by Dunnett's test; NS, not significant; ** $p<0.01$ and ${ }^{* * *} p<0.001$.

\subsection{Macrophage/osteoclast Lineage-Specific Smo Knockout Mice Exhibit Resistance to Age-Related Bone Loss}

Finally, to examine the function of Hh signaling in osteoclasts in vivo, macrophage/osteoclast lineage-specific Smo knockout mice were generated by crossing LysM-Cre mice [29] with Smo-floxed mice [30]. A preliminary microcomputed tomography $(\mu \mathrm{CT})$ analysis revealed that trabecular bone mass decreased significantly with aging in wild-type C57BL/6J mice (Figure 5A). On the other hand, deletion of Smo in the macrophage/osteoclast lineage impaired age-related bone loss (Figure 5B), suggesting that Hh signaling in this lineage is associated with bone reduction with aging. 
A

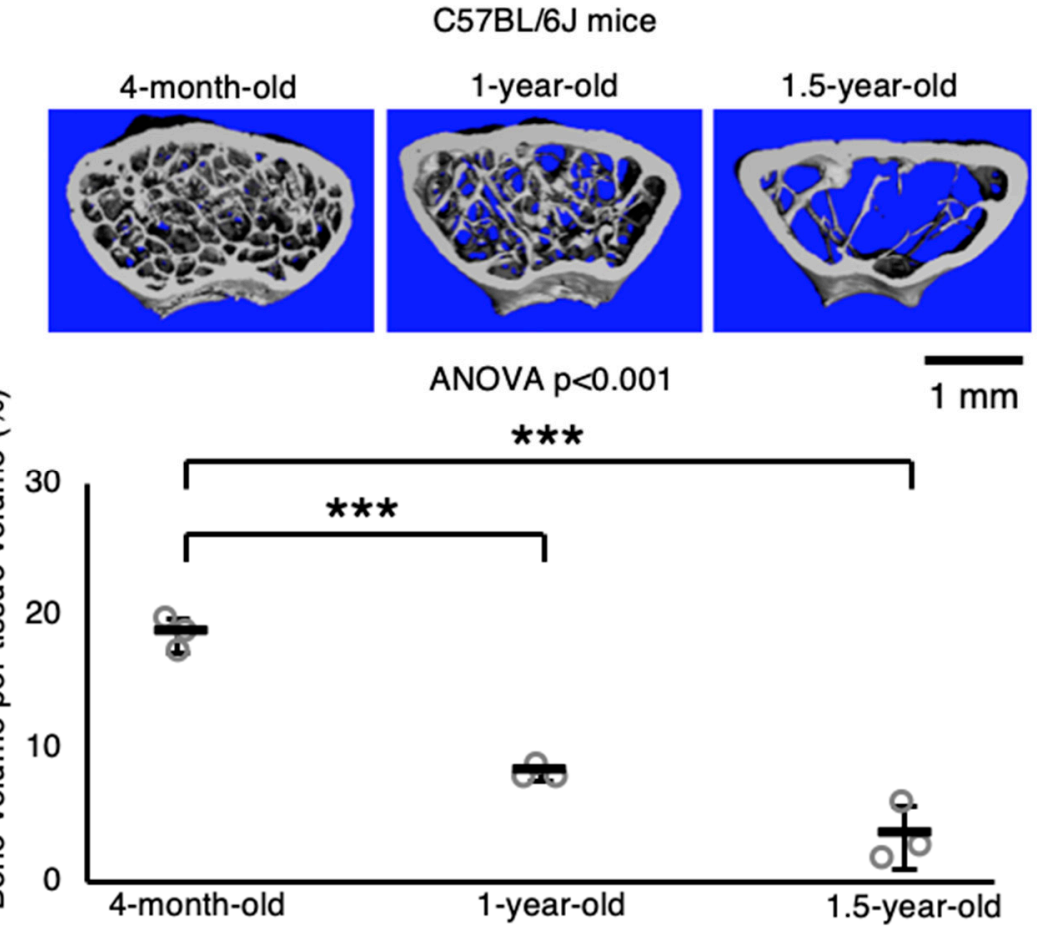

B

$$
\text { LysM-Cre+; Smo flox/flox mice }
$$

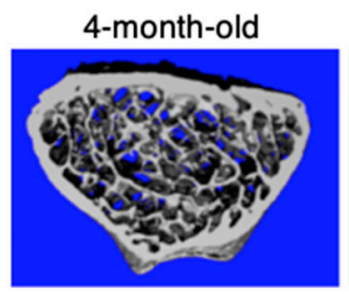

1-year-old

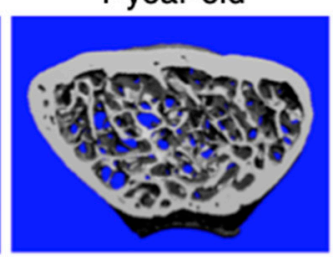

ANOVA $\mathrm{p}=0.18$ 1.5-year-old

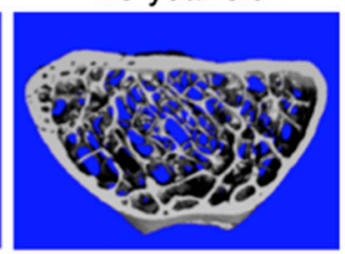

$1 \mathrm{~mm}$

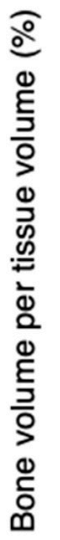

30

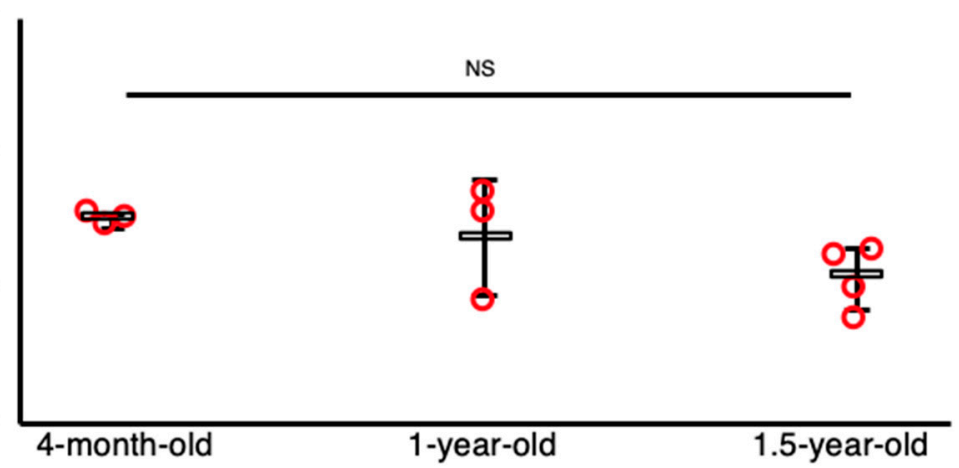

Figure 5. Macrophage/osteoclast lineage-specific Smo knockout mice exhibit resistance to age-related bone loss. (A,B) Distal femurs from (A) C57BL/6J male mice or (B) $\mathrm{LysM}^{-C r e^{+}}$; Smo flox/flox male mice scanned in a Scanco Medical $\mu$ CT 35 System (SCANCO Medical); representative images and three-dimensional bone volume per tissue volume $(\%)(n=3-4)$. Data are represented as means \pm s.d. One-way ANOVA, followed by Dunnett's test; NS, not significant, and ${ }^{* * *} p<0.001$. Note that we separated C57BL/6J mice and $\mathrm{LysM}_{\mathrm{S}} \mathrm{Cre}{ }^{+}$; Smo flox/flox mice datasets, because we maintained C57BL/6J mice and $\mathrm{LysM}-\mathrm{Cre}^{+} ; \mathrm{Smo}{ }^{\text {flox/flox }}$ mice separately. 


\section{Discussion}

This study demonstrates that Hh inhibitors directly function as suppressors of osteoclast formation in in vitro cultures, indicating that Hh signaling in the macrophage/osteoclast lineage is indispensable for osteoclastogenesis (Figure 6A,B). Interestingly, Gli1 mRNA expression, an important indicator of Hh signaling [8], dynamically decreased during the differentiation of pre-osteoclasts (pOCs) into mature osteoclasts (mOCs) (Figure 1C). Additionally, treatment with the GLI1 inhibitor GANT-58 during differentiation of pOCs into mOCs did not affect osteoclast formation in vitro (Figure 3B). These findings indicate that GLI1 is not required for the differentiation of pOCs into mOCs. Taken together with a previous study that oral treatment with cyclopamine suppresses osteoclastic function and results in increased bone mass in mice [27], our findings suggest that Hh signaling inhibitors have a potential as anti-resorptive agents.

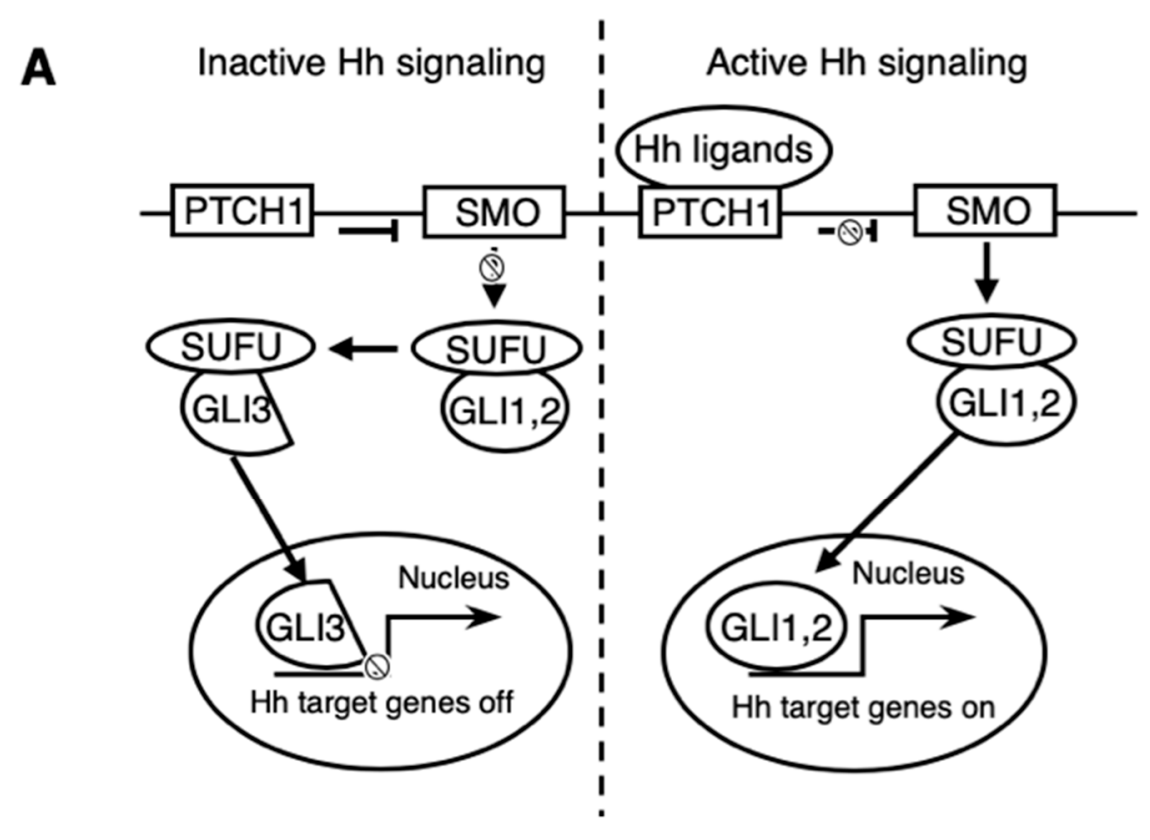

B

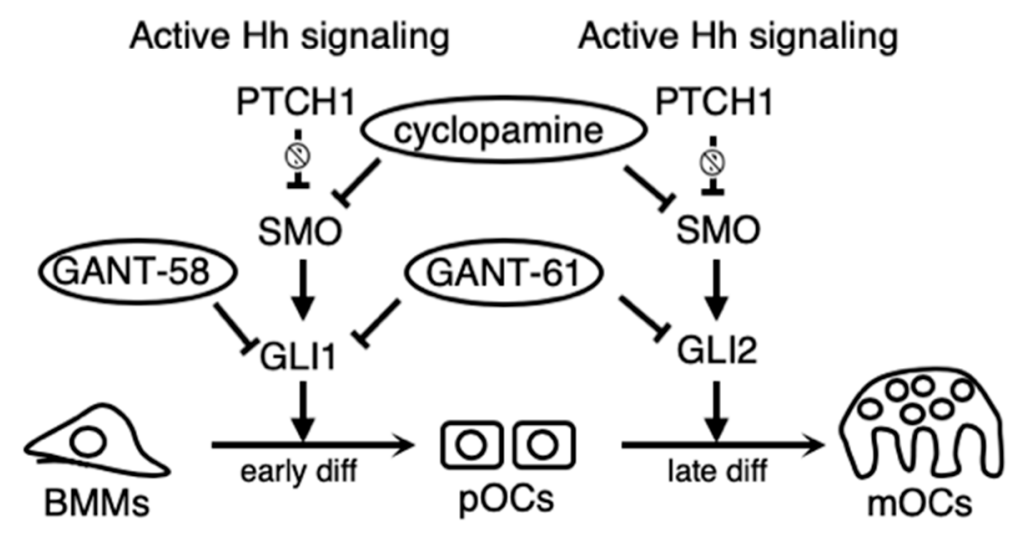

Figure 6. Schema of Hh signaling-mediated osteoclastic differentiation. (A) Schematic of the general Hh-signaling pathway. (B) Proposed model of the newly identified inhibitory mechanism on osteoclastic differentiation by Hh inhibitors. GLI1 activation is a requisite for the differentiation of bone marrow-derived macrophages (BMMs) into pre-osteoclasts (pOCs). GLI2 activation is a requisite for the differentiation of pOCs into mature osteoclasts (mOCs). 
Osteoporosis is a major problem in public health because of its high morbidity and detriment to the quality of life [31]. As aging can be a risk factor in the development of osteoporosis regardless of gender [32], the mechanisms of age-related bone loss are pertinent to the pathogenesis of osteoporosis. Here, our preliminary $\mu \mathrm{CT}$ analysis demonstrated that macrophage/osteoclast lineage-specific deletion of the Smo gene prevents age-related bone loss. As all Hh signaling is transmitted through transmembrane protein SMO, Smo deficiency impairs the transmission of all Hh signaling [33]. Thus, we provide evidence that $\mathrm{Hh}$ signaling in the macrophage/osteoclast lineage is a regulator of age-related bone loss. Ablation of senescent cells pharmacologically or genetically in mice ameliorates age-related bone loss, suggesting that senescence-associated secretory phenotype (SASP) proteins, secreted by senescent cells, account for senile bone loss [34]. Possibly, therefore, Hh ligands may be a kind of SASP protein; nevertheless, no such evidence has been available to date. Since no other hypotheses have been tested, further comprehensive studies are needed to elucidate relevant underlying mechanisms.

In conclusion, this study demonstrates that Hh signaling in the macrophage/osteoclast lineage is requisite for osteoclastogenesis in in vitro cultures and that it mediates age-related bone loss in vivo, indicating the therapeutic potential of Hh inhibitors for senile bone loss.

\section{Materials and Methods}

\subsection{Reagents}

Recombinant M-CSF protein (Cat\# 416-ML-500) was purchased from R\&D Systems, Minneapolis, MN, USA; GST-RANKL (Cat\# 47197900) from Oriental Yeast, Tokyo, Japan; cyclopamine (SMO inhibitor, Cat\# 038-19311) from Wako, Osaka, Japan; GANT-58 (GLI1 inhibitor, Cat\# CS-0507) from Chem Scene, Monmouth, NJ, USA; and GANT-61 (GLI1/2 inhibitor, Cat\# AG-CR1-3561) from AdipoGen, Seoul, Korea.

\subsection{Mice}

C57BL/6J mice were purchased from Clea, Tokyo, Japan. Smo-floxed mouse [30] and LsyM-Cre mouse strains [29] were obtained from Jackson Laboratory, Bar Harbor, ME, USA. All strains were on a C57BL/6J background. All mice were maintained in a specific pathogen-free facility under climate-controlled conditions and a 12-h light/dark cycle and provided with water and standard diet (Oriental Yeast) ad libitum. All animals were handled according to the protocol approved by the Animal Experiment Committee of Ehime University, Japan (Permit No. 05-KU-36-16).

\subsection{Generating M-CSF Overexpression Cells}

To obtain M-CSF overexpression cells, mouse M-CSF cDNA in the pCAG-Neo mIgG2a-Fc vector (Wako) was transfected into mouse fibroblastic LMTK- cells (ATCC, Cat\# CCL-1.3) with X-tremeGENE HP DNA transfection reagent (Roche Diagnostics, Basle, Switzerland). After selection with 5-mg $\mathrm{mL}^{-1}$ G418 (Wako), clones producing high M-CSF-Fc levels were selected by limiting dilution followed by ELISA for Fc-tag protein expression, and the cell culture supernatant of the highest M-CSF-Fc-producing cell line, LMF44-11, was harvested.

\subsection{Osteoclast Culture}

For in vitro osteoclast formation, whole bone marrow cells were harvested from tibias and femurs of eight ten-week-old mice, cultured for three days in $\alpha$-MEM containing antibiotic-antimycotic solution (Gibco, Grand Island, NY, USA) and 10\% FBS supplemented with LMF44-11-conditioned medium used as a source of M-CSF to obtain bone marrow-derived macrophages (BMMs). BMMs were further cultured for four days in medium supplemented with 50-ng mL ${ }^{-1} \mathrm{M}-\mathrm{CSF}$ and 50-ng mL $\mathrm{m}^{-1}$ RANKL. The culture medium was changed every second day. Osteoclasts were identified by TRAP staining or TRAP activity assay, as described in [35,36]. 


\subsection{RNA Isolation and Real-Time RT-PCR}

RNA isolation and real-time RT-PCR were carried out as described in [35,36]. Briefly, total RNA was extracted using TRIzol reagent (Invitrogen, Carlsbad, CA, USA); cDNA was transcribed and used for quantitative RT-PCR conducted with Thunderbird SYBR qPCR Mix (Toyobo Co., Ltd., Osaka, Japan) in the 7500 fast real-time PCR system (Applied Biosystems, Carlsbad, CA, USA). The primers used for RT-PCR are shown in Table S1.

\subsection{MTT Assay}

MTT (3-(4, 5-Dimethylthial-2-yl)-2, 5-Diphenyltetrazalium Bromide) assay was performed as described in [27]. Briefly, BMMs $\left(5 \times 10^{3}\right.$ per well) were plated in 96-well plates with indicated drugs. After $48 \mathrm{~h}$, MTT (Wako) was added for $4 \mathrm{~h}$. $\mathrm{HCl}$ /isopropanol was added to measure absorbance at $570 \mathrm{~nm}$.

\subsection{Microcomputed Tomography}

Microcomputed tomography $(\mu \mathrm{CT})$ scanning of the distal femurs was carried out in a Scanco Medical $\mu$ CT 35 System (SCANCO Medical).

\subsection{Statistical Analysis}

All data are expressed as the means \pm s.d. Statistical analyses were carried out with one-way ANOVA followed by Tukey-Kramer's test or Dunnett's test or unpaired two-sided Student's $t$-test ( $p<0.05,{ }^{* *} p<0.01$, and ${ }^{* * *} p<0.001 ; \mathrm{NS}$, not significant).

Supplementary Materials: Supplementary materials can be found at http://www.mdpi.com/1422-0067/21/8/2745/ s1.

Author Contributions: Y.K. carried out most of the in vitro experiments, analyzed the data, and wrote the manuscript; R.H. designed the study, carried out the animal experiments, analyzed the data, supervised the project, and wrote the manuscript; Y.I. analyzed the data and provided support for the animal experiments; and R.K. and S.K. provided advice on the project and financial support. All authors have read and agreed to the published version of the manuscript.

Funding: This study was supported in part by JSPS KAKENHI, grant numbers JP17K18438 (to Y.K.), JP19K18514 (to Y.K.), and JP18K06832 (to R.H.), and by the JSBMR Rising Star Grant for fiscal year 2018 (to R.H.).

Acknowledgments: We thank Y. Takaoka, C. Shiraishi, Y. Utsunomiya, K. Shimazu, M. Nagao, M. Hashimoto, and H. Iwata for valuable assistance. We also thank G. Yamada (Wakayama Medical University) for his helpful discussion.

Conflicts of Interest: The authors declare no conflicts of interests.

\section{References}

1. Bechtold, T.E.; Kurio, N.; Nah, H.D.; Saunders, C.; Billings, P.C.; Koyama, E. The Roles of Indian Hedgehog Signaling in TMJ Formation. Int. J. Mol. Sci. 2019, 20, 6300. [CrossRef]

2. Haraguchi, R.; Kitazawa, R.; Kohara, Y.; Ikedo, A.; Imai, Y.; Kitazawa, S. Recent Insights into Long Bone Development: Central Role of Hedgehog Signaling Pathway in Regulating Growth Plate. Int. J. Mol. Sci. 2019, 20, 5840. [CrossRef] [PubMed]

3. Hyuga, T.; Alcantara, M.; Kajioka, D.; Haraguchi, R.; Suzuki, K.; Miyagawa, S.; Kojima, Y.; Hayashi, Y.; Yamada, G. Hedgehog Signaling for Urogenital Organogenesis and Prostate Cancer: An Implication for the Epithelial-Mesenchyme Interaction (EMI). Int. J. Mol. Sci. 2019, 21, 58. [CrossRef] [PubMed]

4. Petrova, R.; Joyner, A.L. Roles for Hedgehog signaling in adult organ homeostasis and repair. Development 2014, 141, 3445-3457. [CrossRef] [PubMed]

5. Jeng, K.S.; Chang, C.F.; Lin, S.S. Sonic Hedgehog Signaling in Organogenesis, Tumors, and Tumor Microenvironments. Int. J. Mol. Sci. 2020, 21, 758. [CrossRef]

6. Sasai, N.; Toriyama, M.; Kondo, T. Hedgehog Signal and Genetic Disorders. Front. Genet. 2019, 10, 1103. [CrossRef] 
7. Kong, J.H.; Siebold, C.; Rohatgi, R. Biochemical mechanisms of vertebrate hedgehog signaling. Development 2019, 146. [CrossRef]

8. Varjosalo, M.; Taipale, J. Hedgehog: Functions and mechanisms. Genes Dev. 2008, 22, 2454-2472. [CrossRef]

9. Wang, C.; Pan, Y.; Wang, B. Suppressor of fused and Spop regulate the stability, processing and function of Gli2 and Gli3 full-length activators but not their repressors. Development 2010, 137, 2001-2009. [CrossRef]

10. Zhang, Z.; Shen, L.; Law, K.; Zhang, Z.; Liu, X.; Hua, H.; Li, S.; Huang, H.; Yue, S.; Hui, C.C.; et al. Suppressor of Fused Chaperones Gli Proteins to Generate Transcriptional Responses to Sonic Hedgehog Signaling. Mol. Cell. Biol. 2017, 37. [CrossRef]

11. Lauth, M.; Bergstrom, A.; Shimokawa, T.; Toftgard, R. Inhibition of GLI-mediated transcription and tumor cell growth by small-molecule antagonists. Proc. Natl. Acad. Sci. USA 2007, 104, 8455-8460. [CrossRef]

12. Sabol, M.; Trnski, D.; Musani, V.; Ozretic, P.; Levanat, S. Role of GLI Transcription Factors in Pathogenesis and Their Potential as New Therapeutic Targets. Int. J. Mol. Sci. 2018, 19, 2562. [CrossRef]

13. Agyeman, A.; Jha, B.K.; Mazumdar, T.; Houghton, J.A. Mode and specificity of binding of the small molecule GANT61 to GLI determines inhibition of GLI-DNA binding. Oncotarget 2014, 5, 4492-4503. [CrossRef]

14. Chen, J.K.; Taipale, J.; Cooper, M.K.; Beachy, P.A. Inhibition of Hedgehog signaling by direct binding of cyclopamine to Smoothened. Genes Dev. 2002, 16, 2743-2748. [CrossRef]

15. Incardona, J.P.; Gaffield, W.; Kapur, R.P.; Roelink, H. The teratogenic Veratrum alkaloid cyclopamine inhibits sonic hedgehog signal transduction. Development 1998, 125, 3553-3562.

16. Carballo, G.B.; Honorato, J.R.; de Lopes, G.P.F.; Spohr, T. A highlight on Sonic hedgehog pathway. Cell Commun. Signal 2018, 16, 11. [CrossRef]

17. Hallett, S.A.; Ono, W.; Ono, N. Growth Plate Chondrocytes: Skeletal Development, Growth and Beyond. Int. J. Mol. Sci. 2019, 20, 6009. [CrossRef]

18. Komori, T. Molecular Mechanism of Runx2-Dependent Bone Development. Mol. Cells 2020, 43, 168-175. [CrossRef]

19. Rodda, S.J.; McMahon, A.P. Distinct roles for Hedgehog and canonical Wnt signaling in specification, differentiation and maintenance of osteoblast progenitors. Development 2006, 133, 3231-3244. [CrossRef]

20. Mak, K.K.; Bi, Y.; Wan, C.; Chuang, P.T.; Clemens, T.; Young, M.; Yang, Y. Hedgehog signaling in mature osteoblasts regulates bone formation and resorption by controlling PTHrP and RANKL expression. Dev. Cell 2008, 14, 674-688. [CrossRef]

21. Ohba, S.; Kawaguchi, H.; Kugimiya, F.; Ogasawara, T.; Kawamura, N.; Saito, T.; Ikeda, T.; Fujii, K.; Miyajima, T.; Kuramochi, A.; et al. Patched1 haploinsufficiency increases adult bone mass and modulates Gli3 repressor activity. Dev. Cell 2008, 14, 689-699. [CrossRef]

22. Johnson, R.W.; Nguyen, M.P.; Padalecki, S.S.; Grubbs, B.G.; Merkel, A.R.; Oyajobi, B.O.; Matrisian, L.M.; Mundy, G.R.; Sterling, J.A. TGF-beta promotion of Gli2-induced expression of parathyroid hormone-related protein, an important osteolytic factor in bone metastasis, is independent of canonical Hedgehog signaling. Cancer Res. 2011, 71, 822-831. [CrossRef]

23. Karner, C.M.; Long, F. Glucose metabolism in bone. Bone 2018, 115, 2-7. [CrossRef]

24. Sims, N.A.; Martin, T.J. Coupling Signals between the Osteoclast and Osteoblast: How are Messages Transmitted between These Temporary Visitors to the Bone Surface? Front. Endocrinol. (Lausanne) 2015, 6, 41. [CrossRef]

25. Compston, J.E.; McClung, M.R.; Leslie, W.D. Osteoporosis. Lancet 2019, 393, 364-376. [CrossRef]

26. Harvey, N.C.; McCloskey, E.; Kanis, J.A.; Compston, J.; Cooper, C. Bisphosphonates in osteoporosis: NICE and easy? Lancet 2017, 390, 2243-2244. [CrossRef]

27. Heller, E.; Hurchla, M.A.; Xiang, J.; Su, X.; Chen, S.; Schneider, J.; Joeng, K.S.; Vidal, M.; Goldberg, L.; Deng, H.; et al. Hedgehog signaling inhibition blocks growth of resistant tumors through effects on tumor microenvironment. Cancer Res. 2012, 72, 897-907. [CrossRef]

28. Shimo, T.; Matsumoto, K.; Takabatake, K.; Aoyama, E.; Takebe, Y.; Ibaragi, S.; Okui, T.; Kurio, N.; Takada, H.; Obata, K.; et al. The Role of Sonic Hedgehog Signaling in Osteoclastogenesis and Jaw Bone Destruction. PLoS ONE 2016, 11, e0151731. [CrossRef]

29. Postic, C.; Shiota, M.; Niswender, K.D.; Jetton, T.L.; Chen, Y.; Moates, J.M.; Shelton, K.D.; Lindner, J.; Cherrington, A.D.; Magnuson, M.A. Dual roles for glucokinase in glucose homeostasis as determined by liver and pancreatic beta cell-specific gene knock-outs using Cre recombinase. J. Biol. Chem. 1999, 274, 305-315. [CrossRef] 
30. Long, F.; Zhang, X.M.; Karp, S.; Yang, Y.; McMahon, A.P. Genetic manipulation of hedgehog signaling in the endochondral skeleton reveals a direct role in the regulation of chondrocyte proliferation. Development 2001, 128, 5099-5108.

31. Sirufo, M.M.; Suppa, M.; Ginaldi, L.; De Martinis, M. Does Allergy Break Bones? Osteoporosis and Its Connection to Allergy. Int. J. Mol. Sci. 2020, 21, 712. [CrossRef]

32. Pignolo, R.J.; Samsonraj, R.M.; Law, S.F.; Wang, H.; Chandra, A. Targeting Cell Senescence for the Treatment of Age-Related Bone Loss. Curr. Osteoporos. Rep. 2019, 17, 70-85. [CrossRef]

33. Madhala-Levy, D.; Williams, V.C.; Hughes, S.M.; Reshef, R.; Halevy, O. Cooperation between Shh and IGF-I in promoting myogenic proliferation and differentiation via the MAPK/ERK and PI3K/Akt pathways requires Smo activity. J. Cell. Physiol. 2012, 227, 1455-1464. [CrossRef]

34. Farr, J.N.; Xu, M.; Weivoda, M.M.; Monroe, D.G.; Fraser, D.G.; Onken, J.L.; Negley, B.A.; Sfeir, J.G.; Ogrodnik, M.B.; Hachfeld, C.M.; et al. Targeting cellular senescence prevents age-related bone loss in mice. Nat. Med. 2017, 23, 1072-1079. [CrossRef]

35. Kohara, Y.; Haraguchi, R.; Kitazawa, R.; Kitazawa, S. Knockdown of Lrp1 in RAW264 cells inhibits osteoclast differentiation and osteoclast-osteoblast interactions in vitro. Biochem. Biophys. Res. Commun. 2020, 523, 961-965. [CrossRef]

36. Matsuoka, K.; Kohara, Y.; Naoe, Y.; Watanabe, A.; Ito, M.; Ikeda, K.; Takeshita, S. WAIF1 Is a Cell-Surface CTHRC1 Binding Protein Coupling Bone Resorption and Formation. J. Bone Miner. Res. 2018, 33, 1500-1512. [CrossRef]

(C) 2020 by the authors. Licensee MDPI, Basel, Switzerland. This article is an open access article distributed under the terms and conditions of the Creative Commons Attribution (CC BY) license (http://creativecommons.org/licenses/by/4.0/). 